\section{ORIGINAL RESEARCH}

J. Berge

A. Biondi

P. Machi

H. Brunel

L. Pierot

J. Gabrillargues

K. Kadziolka

X. Barreau

V. Dousset

A. Bonafé

\title{
Flow-Diverter Silk Stent for the Treatment of Intracranial Aneurysms: 1-year Follow-Up in a Multicenter Study
}

BACKGROUND AND PURPOSE: FD stent placement is a promising therapy for challenging intracranial aneurysms. Long-term evaluations about angiographic and morphologic results are still missing. This is the aim of this multicenter series.

MATERIALS AND METHODS: We report our experience and 1-year FU in a retrospective chart review of 65 consecutive subjects with 77 unruptured or recanalized aneurysms that were treated with Silk FD stents at 6 centers in France. Both angiographic and clinical results were recorded before treatment and at 6 and 12 months after treatment. At the 12-month FU, relationships between angiographic aneurysm occlusion and shrinkage of the thrombosed aneurysm sac were evaluated.

RESULTS: Stent deployment was achieved in 64 cases (98.5\%) and failed in 1 case (1.5\%). Seven misdeployments of the Silk stent caused the occlusion of 6 parent arteries. Overall acute/subacute procedural morbidity was $7.7 \%$, and mortality was zero. Delayed complications were observed in $10.9 \%$ of subjects. At the 6 -month FU, permanent morbidity was $7.8 \%$ and mortality was $3 \%$. Complete occlusion occurred within 6 months in $68 \%$ of aneurysms and within 12 months after treatment in $84.5 \%$ of aneurysms. At the 12-month FU, in angiographically complete occluded aneurysms, MR imaging/CT analysis showed the complete disappearance of the thrombosed aneurysm in $30 \%$ of cases and partial shrinkage in $52 \%$; furthermore, thrombosed aneurysms were stable in $11 \%$ of cases and enlarged in $7 \%$.

CoNCLUSIONS: The Silk stent is an effective tool for the treatment of challenging aneurysms because it allows complete occlusion in most cases 1 year after treatment. Permanent morbidity was $7.8 \%$, and mortality was $3 \%$.

ABBREVIATIONS: $\mathrm{ACA}=$ anterior cerebral artery; $\mathrm{CCF}=$ carotid cavernous fistula; $F$ D = flowdiverter; FU = follow-up; ISUIA = International Study of Unruptured Intracranial Aneurysms; $\mathrm{mRS}=$ modified Rankin Scale; PAO = parent artery occlusion; PCA = posterior cerebral artery

$\mathbf{T}$ he advent of FD stents permits an alternative for the treatment of giant/large-neck aneurysms or dissecting/fusiform aneurysms, in which selective endovascular treatment is not possible or is contraindicated, or PAO is not tolerated.

The Silk stent (Balt Extrusion, Montmorency, France) is braided with 48 high-attenuation nickel and titanium alloy wires with 4 platinum markers. The aim of this tight mesh is to divert blood flow from the aneurysm sac toward the downstream artery. If successful, the Silk stent causes a rapid flow reduction and the subsequent thrombosis of the aneurysm sac, as demonstrated in experimental bench-test flow analysis ${ }^{1}$ and in animal studies. ${ }^{2}$ Since 2007, intracranial FD stents have been used in clinical practice for particular configurations of intracranial aneurysms. ${ }^{3-5}$ In regard to large-neck aneurysms, the combination of coils and a stent in the parent artery re-

Received June 5, 2011; accepted after revision September 8.

From the Departments of Neuroradiology, University Hospital, Bordeaux (J.B., X.B., V.D.), Besançon (A.Bi.), Montpellier (P.M., A.Bo.), Marseille (H.B.), Reims (L.P., K.K.), and Clermont-Ferrand (J.G.), France.

Please address correspondence to Jérôme Berge, MD, Service de Neuroradiologie, Hôpital Pellegrin Tripode, Place Amélie Raba-Léon, 33076 Bordeaux Cedex, France: e-mail; jerome. berge@chu-bordeaux.fr

Indicates open access to non-subscribers at www.ajnr.org

Indicates article with supplemental on-line table.

http://dx.doi.org/10.3174/ajnr.A2907

duces the recanalization rate with an increased morbidity. ${ }^{6-8}$ Here, we retrospectively review a consecutive series of subjects with intracranial aneurysms treated with Silk stents in 6 French medical centers. Currently, 1-year FU studies of a large number of intracranial aneurysms treated by using Silk FD stents have not been detailed within the literature, to our knowledge. The aim was to evaluate data concerning the relationships between angiographic aneurysm occlusion and shrinkage of the thrombosed aneurysm sac after Silk stent placement.

\section{Materials and Methods}

We performed a retrospective review of the clinical and radiologic records of all consecutive subjects in a registry of subjects treated with Silk stents at 6 French centers from September 2008 to December 2009. Two operators (J.B. and A.Bi.) collected and reviewed all data independently. All subjects were required to provide their informed consent before the procedure and for chart review. This observational study was approved by our local ethics committee.

\section{Patient and Aneurysm Characteristics}

This study included 65 subjects with 77 intracranial aneurysms; of the aneurysms, 66 were unruptured and 11 were recurrent. The recurrent aneurysms were previously ruptured and treated with coils, or coils and stent placement in 4 subjects. Overall, 58 subjects presented with only 1 aneurysm, while 7 subjects had multiple adjacent lesions. In the 


\begin{tabular}{lc}
\hline Table 1: Patient demographics: $\mathbf{6 5}$ subjects with 77 aneurysms \\
\hline Demographic Data & Values \\
(No.) $(\%)$ \\
\hline Total no. of subjects & 65 \\
$\quad$ Female & $51(78 \%)$ \\
Male & $14(22 \%)$ \\
Average age (yr) & $54($ range, 21-84) \\
Unruptured aneurysms & $66(87 \%)$ \\
Recanalized aneurysms & $11(13 \%)$ \\
Aneurysm morphology & \\
Saccular & $52(68 \%)$ \\
Fusiform or dissecting & $25(32 \%)$ \\
Aneurysm location & \\
ICA extradural & $33(43 \%)$ \\
ICA intradural & $31(40 \%)$ \\
MCA & $2(2.5 \%)$ \\
ACA & $2(2.5 \%)$ \\
Vertebrobasilar & $9(12 \%)$ \\
Asymptomatic subjects & $26(40 \%)$ \\
Symptomatic subjects & $39(60 \%)$ \\
Cavernous sinus & $21(32 \%)$ \\
Midbrain & $5(8 \%)$ \\
Optic nerve & $11(17 \%)$ \\
Other & $2(3 \%)$ \\
\hline
\end{tabular}

\begin{tabular}{|c|c|c|c|c|}
\hline \multicolumn{5}{|l|}{77 Included } \\
\hline Aneurysms & $<7 \mathrm{~mm}$ & $7-12 \mathrm{~mm}$ & $13-24 \mathrm{~mm}$ & $>24 \mathrm{~mm}$ \\
\hline Cavernous carotid & 4 & 3 & 10 & 12 \\
\hline ICA/MCA/ACA & 10 & 8 & 17 & 4 \\
\hline Vertebrobasilar & 0 & 4 & 3 & 2 \\
\hline
\end{tabular}

subjects with multiple aneurysms, 4 subjects had 2 aneurysms, 2 had 3 , and 1 had 5 .

Patient demographic data and aneurysm location and size are detailed in Table 1; aneurysm distribution is detailed in Table 2. Clinical status was assessed by using the mRS. Before the procedure, 27 subjects $(41.5 \%)$ had an mRS score of 0 , nine subjects (14\%) had an mRS score of 1 , and 29 subjects ( $44.5 \%$ ) had an mRS score of 2 . CT scans or MR images or both obtained before the Silk stent procedure were available for all subjects.

\section{Periprocedural Aspects}

All procedures were performed by a senior interventional neuroradiologist with experience in stent-placement techniques. Each operator was assisted by the presence of a proctor during the first 5 patients to discuss the rationale for the use of an artery-reconstruction device and to select the device size; proctors were also present during the deployment phase of the procedure. In all subjects, aneurysm treatment was performed with the patient under general anesthesia. Parent artery measurements were performed for the selection of the Silk stent size; for this, both distal and proximal artery diameters in the landing zone of the stent were measured.

In total, 73 Silk stents were used in 65 subjects with 77 aneurysms; subjects received $\geq 1$ Silk stent. Fifty-one subjects with 1 aneurysm were treated by using 1 stent; 6 subjects, with 2 stents; and 1, with 3 stents. However, 7 subjects with multiple adjacent aneurysms were treated with only 1 stent. Overall, coils (previous and/or added during FD stent placement) were present in 16 (21\%) aneurysms (6 recurrent aneurysms with previous coils and 4 with both previous and added coils, and 6 with added coils during FD procedure). During the Silk

\begin{tabular}{|c|c|c|}
\hline & $\begin{array}{c}\text { 6-Month } \\
\text { FU }\end{array}$ & $\begin{array}{c}\text { 1-Year } \\
\text { FU }\end{array}$ \\
\hline \multicolumn{3}{|l|}{ Angiographic Results } \\
\hline Stenting failure & $1(1 \%)$ & $1(1 \%)$ \\
\hline Mortality & $2(4 \%)$ & $2(3 \%)$ \\
\hline Aneurysms unavailable for FU or FU pending & $18(32 \%)$ & $4(6 \%)$ \\
\hline Total & $21(37 \%)$ & $7(10 \%)$ \\
\hline Evaluated aneurysms & 56 & 70 \\
\hline Residual aneurysm & $11(20 \%)$ & $3(4 \%)$ \\
\hline Residual neck & $3(5 \%)$ & $4(6 \%)$ \\
\hline Complete occlusion & $38(68 \%)$ & $59(84 \%)$ \\
\hline PAO & $4(7 \%)$ & $4(6 \%)$ \\
\hline
\end{tabular}

stent procedure, coils were added in 10 (13\%) aneurysms ( 4 recurrent and 6 de novo aneurysms). In total, 51 aneurysms had no coils. Flow modification after Silk implantation was classified following the scale of Szikora et al, ${ }^{3}$ as either complete stasis or significant flow reduction or slow flow.

\section{Antiplatelet and Other Drug Regimens}

Antiplatelet therapy was established for all subjects receiving a Silk stent. The protocol was the same one used for other intracranial stents and was as follows: From days 3-7 before the procedure and for 2-3 months after the procedure, subjects were given a dosage of $75 \mathrm{mg}$ per day of clopidogrel in conjunction with 160-250 mg of aspirin, depending on the center. During the procedure, a heparin bolus (50 $\mathrm{UI} / \mathrm{kg}$ ) and nimodipine were administered according to need. Responsiveness tests to clopidogrel and aspirin were not available at all of the centers.

\section{Follow-Up}

Six-month and 1-year angiographic FU were performed by using the Montreal grading system ${ }^{9}$ with 3 grades: complete occlusion (class 1 ), residual neck (class 2), and residual aneurysm (class 3) (Table 3). At the 6-month FU, 56 aneurysms were assessed; a total of 70 were assessed for 1-year angiographic FU. At the 1-year FU, CT scans and/or MR images were available in 54 subjects with 66 aneurysms (Table 4). To evaluate thrombosed aneurysm sac shrinkage, all lesions were measured on preprocedural CT scans or MR images in the axial plane with 2 orthogonal measurements and were compared with the FU examinations. MR imaging protocol was different in each center, but the measurement method of the aneurysm size was the same before and after treatment in each center to ensure reliability of aneurysm size variation. Among 66 aneurysms with FU MR imaging analysis, 9 were treated with FD stents and coils, but 1 of these was lost to FU (Table 4). Intermediate 6-month (58 subjects) and 1-year (58 subjects) clinical FUs were assessed by mRS.

\section{Statistical Analysis}

One year after the procedure, in relation to the shrinkage of the aneurysm sac on an MR imaging study, aneurysms were categorized into 2 groups: 1) disappeared or decreased aneurysms, and 2) increased or unchanged aneurysms. The comparison of variables between these 2 groups was performed by using the nonparametric Fisher exact test. A $P$ value $<.05$ was considered significant. Binary logistic regression was used to verify the lack of influence of aneurysm morphology and volume on regression after treatment. Tanagra 1.4.38 software (http://eric.univ-lyon2.fr/ ricco/tanagra/) was used to perform the logistic regression. 
Table 4: Relationships between aneurysm angiographic occlusion and aneurysm shrinkage visualized in an MR imaging or CT study at 1-year FU in 54 subjects with 66 aneurysms

\begin{tabular}{|c|c|c|c|c|c|}
\hline Result & Residual Aneurysm & Residual Neck & Complete Occlusion & PAO & Total \\
\hline Disappeared & & & $17(30 \%)$ & 1 & $18(27 \%)$ \\
\hline Decreased & 2 & 3 & $29(1)^{\mathrm{a}}(52 \%)$ & 1 & $35(1)^{a}(53 \%)$ \\
\hline Unchanged & & & $6(3)^{a}(11 \%)$ & 1 & $7(3)^{a}(11 \%)$ \\
\hline Increased & & $1(1)^{\mathrm{a}}$ & $4(3)^{\mathrm{a}}(7 \%)$ & $1(1)^{\mathrm{a}}$ & $6(5)^{a}(9 \%)$ \\
\hline Total & $2(3 \%)$ & $4(1)^{a}(6 \%)$ & $56(7)^{a}(85 \%)$ & $4(1)^{a}(6 \%)$ & $66(9)^{a}(100 \%)$ \\
\hline
\end{tabular}

${ }^{a}$ Aneurysms with coils added during the FD stenting procedure.

\section{Results}

\section{Stent Procedure}

Silk stent placement was attempted in 65 subjects with 77 aneurysms. Stent deployment was achieved in 64 cases $(98.5 \%)$. In the 1 failed case $(1.5 \%)$, the microcatheter and the Silk device were removed without consequences. Definitive deployment was obtained for 72/73 stents and 76/77 aneurysms, including 63 single aneurysms treated by multiple stents and 13 multiple adjacent aneurysms treated with a single stent. However, while correct deployment and full stent expansion were observed for 63 stents $(86.3 \%)$, 9 stents $(12.3 \%)$ were incompletely or incorrectly deployed. Misdeployments occurred on curved vessel segments with a sharp angle of $\geq 90^{\circ}$. We attempted to expand 3 incompletely deployed Silk stents by balloon (2 cases) or the deployment of an Enterprise stent (Cordis, Miami Lakes, Florida) within the Silk stent (1 case). In total, 7 persistent misdeployments caused PAO in 6 subjects (9.3\%); occlusion occurred immediately in 5 subjects and at day 4 in 1 patient.

\section{Immediate Angiographic Results}

According to the classification of Szikora et al, ${ }^{3}$ complete stasis with no contrast material entering the sac was observed in only 5 lesions (6.6\%) excluding 5 others with PAO (6.6\%), while significant flow reduction or slow flow was observed in 66 lesions (92\%).

\section{Acute Complications}

Six acute PAOs resulted in stroke in 4 subjects and were uneventful in 2 subjects (On-line Table 1). In 1 patient with a left MCA giant fusiform aneurysm and occlusion of the parent artery, resistance to clopidogrel was later biologically proved. At day 4, another patient also had a stroke due to Silk thrombosis. Among 5 acute or subacute strokes, 3 developed from a sacciform aneurysm, and 2, from a fusiform aneurysm. Overall acute/subacute procedural morbidity was observed in 5 of 65 subjects (7.7\%); no mortality was observed.

\section{Delayed Complications}

Delayed complications that occurred between 2 weeks and 5 months after the procedure were observed in 7 of $64(10.9 \%)$ subjects (On-Line Table 1). There were 3 ischemic complications ( 1 stroke and 2 TIAs) and 3 delayed ruptures. One month after the procedure, 1 patient experienced a stroke causing a right hemiplegia. In this case, the stent was correctly deployed. Two weeks and 3 months after the procedure, 2 subjects presented with TIAs. Three of these ischemic events occurred at clopidogrel interruption: the first, after the patient decision to stop this treatment on his own (right hemiplegia at day 4), second with onset of right hemiplegia at 1 month and third with TIA at 3 months (right hemianesthesia).

Three delayed ruptures included 1 CCF 14 days after the procedure, successfully treated by the venous route. One subject with an acute stroke due to the ACA experienced aneurysm bleeding at day 135 . Another with MCA + PCA occlusions experienced an aneurysm rupture at day 20 and died. None of the 3 aneurysms with delayed rupture were treated with coils during the FD procedure. In these 3 cases, complete occlusion of the aneurysm sac was documented either by angiogram or MR angiography. Additionally, at 1 month, 1 patient died because of severe hemorrhage during surgical treatment for a previously known malignant meningioma. This tumor rapidly increased in size, also presenting hemorrhagic components caused most likely by the clopidogrel therapy. Excluding the latter patient, bleeding from the aneurysm was observed in 3 of 76 aneurysms (4\%) and in 3 of 64 subjects $(4.7 \%)$. Consequently, at the 6-month FU, permanent morbidity was observed in 5 of 64 subjects $(7.8 \%)$ and mortality occurred in 2 of 64 subjects (3\%). All severe complications were observed in lesions located in the anterior circulation. A transient worsening occurred during the first 2 weeks after the procedure in 24 of 64 of subjects (37.5\%). Clinically, this consisted of headaches associated with worsening of symptoms due to an increased mass effect in the cavernous sinus (9 cases), optic nerve (3 cases), or brain stem ( 3 cases). This perianeurysmal brain inflammation was treated by corticoid therapy by using decreasing doses for at least 2 weeks. MR imaging usually showed rapid evolutive thrombosis (between $50 \%$ and $100 \%$ of the sac volume), perianeurysmal vasogenic edema, and circumferential enhancement after contrast injection.

\section{Angiographic Follow-Up}

We observed a progressive increase of the aneurysm complete occlusion rate (Table 3 ). Complete angiographic occlusion (class 1 from Roy et $\mathrm{al}^{9}$ ) was present in 38 of 56 aneurysms $(68 \%)$ at the 6-month follow-up and in 59 of 70 aneurysms $(84.3 \%)$ at the 1-year follow-up. Conversely, residual neck and residual aneurysm rates (class 2/3) shifted from 59\% immediately after the procedure to 11 of 56 aneurysms (19.6\%) evaluated at the 6-month follow-up and 3 of 70 aneurysms (4.3\%) evaluated 1-year FU.

No particular morphology/location of the sac or of the aneurysm neck explained the poor occlusion rate in some of the lesions at the follow-up evaluations. No new PAO occurred. However, intrastent stenosis of $\leq 50 \%$ was observed in 5 subjects of 52 with open arteries at the 6-month FU. All subjects were asymptomatic and showed complete recovery of the in- 
trastent parent artery diameter at the 1-year FU. No aneurysm recurrence was observed in these subjects.

\section{MR Imaging/CT 1-Year Follow-Up, and Angiographic Correlations}

At the 1-year FU, the stent-placement procedure failed in 2 subjects, 2 subjects died, 6 subjects were unavailable for evaluation, and 2 subjects had not yet undergone MR imaging/CT scanning (total of 11 subjects with 11 aneurysms). Consequently, CT scans and/or MR images were available for 54 subjects with 66 aneurysms, which allowed the evaluation of the relationship between aneurysm angiographic results and aneurysm shrinkage. CT and/or MR images were not available in 4 of 70 aneurysms. These results are summarized in Table 4.

In 56/66 angiographically complete occluded aneurysms, MR/CT analysis showed the complete disappearance of the thrombosed aneurysm in 17 lesions (30\%) and partial shrinkage of the aneurysm in 29 lesions (52\%). Of the remaining aneurysms, 6 remained unchanged in size (11\%) and $4(7 \%)$ were enlarged. Nine of the 66 analyzed lesions (13.6\%) were also treated with coils during the Silk FD stent placement procedure. No coils were added in 18 angiographically occluded aneurysms that shrank and disappeared. Coils were added in 1 of $35(2.8 \%)$ aneurysms that reduced in size, in 3 of $7(43 \%)$ aneurysms that remained unchanged, and in 5 of $6(83 \%)$ aneurysms that increased in size despite complete angiographic occlusion in 4 cases (Table 4 ).

Statistical analysis of the 2 groups regarding shrinkage of the aneurysm sac in relation to coils added during the FD stent placement included the following: 1) 53 disappeared or decreased aneurysms (only 1 with coils), and 2) 13 increased or unchanged aneurysms ( 8 with coils). This result suggests that additional coils within the aneurysm sac during the FD deployment could prevent aneurysm shrinkage and also promote aneurysm growth $(P<.01)$.

This result was confirmed if all lesions with coils inside the aneurysm sac (previous coils and/or coils added during FD stent placement) were considered. A comparison of 51 aneurysms without coils (49 decreased or disappeared [96\%]) and 2 unchanged or increased) and 15 aneurysms with coils (4 decreased or disappeared [26\%] and 11 unchanged or increased) was also statistically significant $(P<.01)$.

The 1-year angiographic occlusion rate of 7 lesions unchanged in size showed complete occlusion in 6 aneurysms (grade 4) and PAO (grade 4c) in 1 aneurysm, whereas in 6 lesions increased in size, we had complete occlusion in 4 aneurysms, residual neck in 1, and PAO in 1. Analysis of this last subgroup showed that 2 were fusiform and 4 were saccular; 2 were cavernous carotid and 4, supraclinoid aneurysms. Size varied from $6 \times 3 \mathrm{~mm}$ to $29 \times 26 \mathrm{~mm}$, and the percentage of enlargement varied from $10 \%$ to $380 \%$ (respectively, 380\%, 40\%, 250\%, 10\%, 170\%, and 30\%). No correlation with preoperative aneurysm size or location was observed. Binary logistic regression was used to verify the noninfluence of the aneurysm morphology and volume on regression after treatment. Regression analysis confirmed that neither morphology nor volume significantly impacted aneurysm shrinkage.

\section{Clinical Follow-Up}

At the 6-month FU when 58 subjects were evaluated, 42 subjects $(72.4 \%$ ) had an mRS score of 0 (versus $41.5 \%$ of subjects at pretreatment), 6 subjects $(10.3 \%)$ had an mRS score of 1 (versus $14 \%$ of subjects at pretreatment), and 3 subjects (5.1\%) had an mRS score of 2 (versus $44.5 \%$ of subjects at pretreatment). Five strokes resulting in permanent deficits were responsible for 3 subjects (5.1\%) with an mRS score of 3, 1 patient $(1.7 \%)$ with an $\mathrm{mRS}$ score of 4 , and another patient $(1.7 \%)$ with an mRS score of 5 . Two subjects $(3.4 \%)$ died (mRS, 6).

At the 1-year FU, 3 subjects were unavailable for evaluation, and the stent-placement procedure failed in 1 patient. Consequently, 61 of 65 subjects were evaluated. Apart from the 2 deaths (3.2\%), 47 subjects (77\%) had an mRS score of 0 , six subjects $(9.8 \%)$ had an mRS score of 1 , two subjects $(3.2 \%)$ had an mRS score of 2 , two subjects $(3.2 \%)$ had an mRS score of 3 , and 2 subjects (3.2\%) had an mRS score of 4 .

\section{Discussion}

The advent of FD stents offers a new therapeutic option for challenging giant/large-neck aneurysms or recurrent aneurysms. However, precise indicators for the use of these new devices are still uncertain. In the literature, most cases include subjects without any other endovascular or surgical options. ${ }^{3,4,10}$ In our study, indications for Silk FD stent treatment included the following: 1) subjects with an unruptured aneurysm with a 5-year cumulated risk of hemorrhage of $>14.5 \%$ following the ISUIA ${ }^{11}$ criteria (30/77 aneurysms in our study) (Table 2), 2) symptomatic subjects with carotid cavernous (29/77) and carotid ophthalmic (8/77) aneurysms, and 3) subjects with a previously ruptured aneurysm or one that harbored several aneurysms on a dysplastic carotid segment (10/77 aneurysms). In carotid cavernous aneurysms, reasons for choosing FD rather than PAO included no functional circle of Willis or the young age of the patient.

Misdeployment with a poor apposition of the Silk stent is still a main concern. This event was encountered in 9 of 63 stents $(12.3 \%)$. Two of these instances were corrected by the additional use of a balloon or stent. Persistent misdeployment of the Silk stent caused occlusion of the parent artery in $9.3 \%$ of cases. The incidence of acute PAO in the literature is difficult to compare from Silk to Pipeline (Chestnut Medical Technologies, Menlo Park, California) studies because of population heterogeneity, but it seems that PAO is more likely to happen with the Silk stent: $9.3 \%$ in our experience; $10 \%$ in the series of Byrne et $\mathrm{al}^{10}$; and $4 \%$ in the experience of Lubicz et $\mathrm{al}^{12}$ rather than with the Pipeline device: no PAO in the Lylyk et $\mathrm{al}^{4}$ and Nelson et $\mathrm{al}^{5}$ series; and $2.4 \%$ in the series of Szikora et $\mathrm{al}^{3}{ }^{3}$ A curved landing zone and stent oversizing are the main parameters to take into account. ${ }^{13}$ Therefore, accurate preoperative measurements both upstream and downstream of the landing zone are critical to match the Silk stent to the arterial diameter. Stent coverage of perforators or side branch arteries may be responsible for ischemic complications. ${ }^{14}$ In our study, we observed 1 case of a stroke after occlusion of the ACA A1 segment with the origin covered by the stent.

The aims of flow diversion are to allow the rapid progression of thrombosis within the aneurysm sac, to protect the patient from bleeding, and to decrease mass effects related to 
aneurysm pulsatility. This diversion of endosaccular blood flow has been documented by several hemodynamic studies ${ }^{1}$ and in animal models. ${ }^{2}$ The optimal porosity to achieve flow reduction and slowness appears to be $70 \%$ (1\%). However, the FD approach does not provide immediate aneurysm occlusion or subsequent protection from bleeding. In our study, only 5 of 76 aneurysms (7\%) showed complete stasis after the Silk stent procedure, added to 5 other noncirculating aneurysms with concomitant PAO. Despite poor immediate efficacy, our results confirm good midterm efficacy of flow diversion to obtain progressive aneurysm occlusion; excluding aneurysm occlusion with a concomitant PAO, $68 \%$ of aneurysms were completely occluded at 3 or 6 months after treatment. Furthermore, at the 1-year FU, $84.5 \%$ of aneurysms were completely occluded.

This long-term FU is important for investigating whether flow diversion is an efficient technique to obtain definitive aneurysm angiographic occlusion. After FD stent placement, Byrne et $\mathrm{al}^{10}$ and Lylyk et $\mathrm{al}^{4}$ reported that $50 \%$ and $56 \%$ of aneurysms were occluded at the 3-month FU, respectively. Additionally, in 1 study, ${ }^{4}$ the authors found that $95 \%$ of aneurysms were occluded 12 months after treatment. As reported in a separate article, ${ }^{3}$ no aneurysm recurrence has been observed so far in our subjects. Authors ${ }^{7}$ have observed angiographic recurrences in $33.5 \%$ of aneurysms treated only with coils and in $15 \%$ to $20 \%$ of aneurysms treated with stents and coils. ${ }^{6,7}$ Recurrence is also reported in retreated lesions with long-term outcomes. ${ }^{15}$

In our study, acute/subacute procedural morbidity was $7.7 \%$, while mortality was zero. Delayed complications, occurring between 2 weeks and 4 months posttreatment, were observed in 6 of $64(9.4 \%)$ subjects. There were 3 ischemic $(4.7 \%)$ and 4 hemorrhagic $(6.2 \%)$ complications. At the 6-month FU, permanent morbidity was observed in $7.8 \%$ of subjects and mortality, in $3 \%$. In 1 of the 2 deaths, death was related to antiplatelet-therapy discontinuation and not to the Silk stent placement itself. In the literature, ${ }^{5,3,10,12}$ morbidity for FD stent placement for intracranial aneurysms ranged from $4 \%$ to $15 \%$, and mortality ranged from $4 \%$ to $8 \%$. These results in FD stent placement should be compared with those obtained after PAO treatment. Despite the sacrifice of the carotid artery, a recent article ${ }^{16}$ reported excellent long-term results with neither mortality nor permanent morbidity in selected subjects who tolerated the PAO test.

In our data, most cases of ischemic complications were observed in subjects with incorrect Silk stent deployment and subsequent in-stent thrombosis. We only observed a late onset of right hemiplegia related to stent thrombosis in 1 patient with correct stent deployment. In this case, we speculate the involvement of a suboptimal antiplatelet regimen as observed in 2 other subjects presenting with a TIA after the discontinuation of antiplatelet treatment. Ischemic complications highlight the need to better assess the antiplatelet response. ${ }^{17}$

Excluding the subject who fatally hemorrhaged during surgical tumor intervention, aneurysm rupture was observed in 3 subjects $(4.7 \%)$. In all 3 cases, rupture was delayed (days 14, 20 , and 135). Two of our delayed ruptures were reported by Kulcsár et $\mathrm{al}^{18}$ with proved complete occlusion of the sac, documented by angiography, realized before the rupture. In our third bleeding case, occlusion was documented by MRA 1 week before CCF onset. Kulcsár et $\mathrm{al}^{18}$ showed that most bleeding after FD stent placement (12 of 13) occurred in partially or completely thrombosed aneurysms with a variable time interval after stent deployment from day 2 to 4 months. Acute bleeding may also occur in the case of stent migration into the sac. ${ }^{12}$ In a case report, ${ }^{19}$ a rupture occurred at day 20 in an almost completely occluded aneurysm after transient visual impairment was treated with steroids. In our 3 cases of delayed rupture, angiographic control before the hemorrhage showed that the aneurysm was completely occluded.

Postmortem examination findings were reported ${ }^{18}$ in 2 subjects who died from delayed hemorrhaging after FD stent placement. The rerupture site was reported at the persistent permeable aneurysm sac or at the site of recanalization. We did not observe delayed bleeding of aneurysms treated with a Silk stent and coils; however, this finding is not significant because in our study, only $13 \%$ of aneurysms were treated with coils in addition to a Silk stent. Despite changes in the March 2010 instructions for use by the manufacturer, suggesting that the Silk stent should be used in association with intrasaccular coils, we think that this assumption is not based on reliable results. In another study, ${ }^{3}$ no difference was observed regarding bleeding in aneurysms treated by an FD stent alone or an FD stent and coils. In a recent article, ${ }^{20}$ the authors reported that intra-aneurysmal pressure increased after FD stent deployment; furthermore, they suggest that the placement of coils or other prothrombotic devices before FD stent deployment could more rapidly achieve aneurysm thrombosis and minimize the time for hemorrhage risk.

The indication for FDs in the acute bleeding phase is unclear, given the necessity of a double antiplatelet therapy and the delayed efficiency of FD stents. At the present time, it should not be the first treatment option for ruptured aneurysms. The association of FDs and coils within the sac may be an option and needs further study to evaluate efficiency in preventing new ruptures and to establish the reliability of flow disappearance within the sac.

In our study, transient asymptomatic intrastent stenosis was observed in 5 of 64 subjects (7.8\%). In the literature, significant parent artery stenosis at 6 months was reported in $\leq 33 \%$ of cases. $^{15}$

Here, we report a transient worsening in 24 of 64 subjects $(37.5 \%)$ due to perianeurysmal brain inflammation. Headache was a constant symptom, sometimes associated with neurologic symptoms. This complication has been described after PAO for giant carotid cavernous aneurysms, ${ }^{21}$ after endovascular aneurysm treatment by using active coils, ${ }^{22}$ and recently after the use of FD stents. ${ }^{23}$

\section{Follow-Up}

In our study, we report the progressive occlusion of aneurysms following Silk FD stent deployment. It is likely that persistent dual antiplatelet therapy in the first months after FD stent deployment could prevent complete thrombosis. There are no data in the literature concerning the relationship between flow reduction and aneurysm shrinkage after FD stent therapy, to our knowledge. As observed in carotid cavernous aneurysms after PAO, ${ }^{6}$ retraction is a key factor for mass-effect reduction and the possible recovery from oculomotor nerve palsy. Complete angiographic occlusion is not always associated with the 
regression or shrinkage of the aneurysm sac itself. Among 56 angiographically complete occluded aneurysms observed 1 year after treatment, 46 lesions $(82 \%)$ decreased or disappeared compared with $10(18 \%)$ aneurysms that remained unchanged or increased. The presence of coils can impair aneurysm shrinkage. Our morphologic evaluation showed that $96 \%$ of aneurysms without coils inside the sac decreased or disappeared compared with only $26 \%$ of aneurysms with coils. These results were validated by statistical analysis $(P<.01)$. On the basis of these data, coils within the aneurysm sac during the FD deployment could prevent aneurysm shrinkage while also promoting aneurysm growth. Therefore, the use of coils is not suggested to achieve a complete cure of the aneurysm sac defined by the association of an angiographic occlusion and complete sac shrinkage. However, all of the cases of delayed aneurysm rupture in the series of Kulcsár et $\mathrm{al}^{18}$ were treated with a Silk stent alone except 1 treated with additional very loose coil packing, while all of our 3 delayed ruptures had no additional coils within the sac. Thus, Balt recommends the use of the associated coils to prevent delayed aneurysm rupture in an alert from the French sanitary security agency in March 2010.

\section{Limitations}

Our study is retrospective in nature and is consequently associated with limitations of such a study. The evaluation of systematic antiplatelet response during FU is lacking. Most likely, we have underestimated the percentage of subjects resistant to antiplatelet medication because only 1 patient who had a stroke exhibited documented resistance to clopidogrel. It would have been of interest to correlate pre-existing intraaneurysmal thrombus and further posttreatment enlargement, but these data were not available in our retrospective study. Regarding Silk FD misdeployments in the carotid siphon, PAO has to be discussed whenever an occlusion test is successful, especially in carotid cavernous aneurysms.

\section{Conclusions}

Flow diversion is a promising technique for the treatment of challenging intracranial aneurysms. The Silk stent induces progressive aneurysm occlusion with complete angiographic occlusion and a patent parent artery in $84 \%$ of subjects at 1 year after treatment. Despite complete angiographic occlusion, the disappearance of the thrombosed aneurysm sac was observed in only $30 \%$ of cases, and shrinkage, in another $52 \%$. As expected, coils within the aneurysm sac appear to prevent aneurysm shrinkage and promote aneurysm growth after FD stent deployment. Rigorous monitoring of antiplatelet treatment and correct FD stent deployment are mandatory to limit ischemic complications. The risk of delayed aneurysm bleeding after FD Silk stent use exists (4.7\% in our series) and highlights the need for a better understanding of its mechanisms. More experience is needed to further refine the possible indicators and the technical aspects of this treatment to improve clinical results.

\section{Acknowledgments}

We thank the following colleagues for including additional subjects in the data base: Emmanuel Chabert, Clermont-
Ferrand (2 cases); Betty Jean, Paris (3 cases); and Nader Sourour, Paris (1 case).

Disclosures: Alessandra Biondi-UNRELATED: Consultancy. Stryker (France). Laurent Pierot-UNRELATED: Consultancy. ev3, MicroVention, Stryker.

\section{References}

1. Lieber Baruch B, Stancampiano AP, et al. Alteration of hemodynamics in aneurysm models by stenting: influence of stent porosity. Ann Biomed Eng 1997;25:460-69

2. Sadasivan C, Cesar L, Seong J, et al. An original flow diversion device for the treatment of intracranial aneurysms: evaluation in the rabbit elastase-induced model. Stroke 2009;40:952-58

3. Szikora I, Berentei Z, Kulcsar Z, et al. Treatment of intracranial aneurysms by functional reconstruction of the parent artery: the Budapest experience with the Pipeline embolization device. AJNR Am J Neuroradiol 2010;31:1139-47

4. Lylyk P, Miranda C, Ceratto R, et al. Curative endovascular reconstruction of cerebral aneurysms with the Pipeline embolization device: the Buenos Aires experience. Neurosurgery 2009;64:632-42, discussion 642-43

5. Nelson PK, Lylyk P, Szikora I, et al. The Pipeline Embolization Device for the Intracranial Treatment of Aneurysms Trial. AJNR Am J Neuroradiol 2011;32: 34-40. Epub 2010 Dec 9

6. Biondi A, Janardha V, Katz JM, et al. Neuroform stent-assisted coil embolization of wide-neck intracranial aneurysms: strategies in stent deployment and midterm follow up. Neurosurgery 2007;61:460-68

7. Piotin M, Blanc R, Spelle L, et al. Stent-assisted coiling of intracranial aneurysms: clinical and angiographic results in 216 consecutive aneurysms. Stroke 2010;41:110-15

8. Maldonado IL, Machi P, Costalat V, et al. Neuroform stent-assisted coiling of unruptured intracranial aneurysms: short- and midterm results from a single-center experience with 68 patients. AJNR Am J Neuroradiol. 2011;32:13136. Epub 2010 Oct 21

9. Roy D, Milot G, Raymond J. Endovascular treatment of unruptured aneurysms. Stroke 2001;32:1998-2004

10. Byrne JV, Beltechi R, Yarnold J, et al. Early experience in the treatment of intra-cranial aneurysms with the Silk flow diverter: procedural and short term outcomes. PLoS One 2010;5: e12492

11. Wiebers DO, Whisnant JP, Huston J 3rd, et al, for the International Study of Unruptured Intracranial Aneurysms Investigators. Unruptured intracranial aneurysms: natural history, clinical outcome, and risks of surgical and endovascular treatment. Lancet 2003;362:103-10

12. Lubicz B, Collignon L, Raphaeli G, et al. Flow-diverter stent for the endovascular treatment of intracranial aneurysms: a prospective study in 29 patients with 34 aneurysms. Stroke 2010;41:2247-53

13. Aurboonyawat T, Blanc R, Schmidt P, et al. An in vitro study of Silk stent morphology. Neuroradiology 2011;53:659-67. Epub 2010 Oct 28

14. Kulcsár Z, Ernemann U, Wetzel SG, et al. High-profile flow diverter (Silk) implantation in the basilar artery: efficacy in the treatment of aneurysms and the role of the perforators. Stroke 2010;41:1690-96

15. Ries T, Siemonsen S, Thomalla G, et al. Long-term follow-up of cerebral aneurysms after endovascular therapy prediction and outcome of retreatment. AJNR Am J Neuroradiol 2007 28:1755-61

16. Clarençon F, Bonneville F, Boch AL, et al. Parent artery occlusion is not obsolete in giant aneurysms of the ICA: experience with very-long-term follow-up. Neuroradiology 2011;53:873-82

17. Anderson CD, Biffi A, Greenberg SM, et al. Personalized approaches to clopidogrel therapy: are we there yet? Stroke 2010;41:2997-3002

18. Kulcsár Z, Houdart E, Bonafé A, et al. Intra-aneurysmal thrombosis as a possible cause of delayed aneurysm rupture after flow diversion treatment. AJNR Am J Neuroradiol 2011;32:20-25. Epub 2010 Nov 11

19. Turowski B, Macht S, Kulcsár Z, et al. Early fatal hemorrhage after endovascular cerebral aneurysm treatment with a flow diverter (Silk stent): do we need to rethink our concepts? Neuroradiology 2011;53:37-41. Epub 2010 Mar 26

20. Cebral JR, Mut F, Rasch M, et al. Aneurysm rupture following treatment with flow-diverting stents: computational hemodynamics analysis of treatment. AJNR Am J Neuroradiol 2011:32;27-33. Epub 2010 Nov 11

21. Hammoud D, Gailloud P, Olivi A, et al. Acute vasogenic edema induced by thrombosis of a giant intracranial aneurysm: a cause of pseudostroke after therapeutic occlusion of the parent vessel. AJNR Am J Neuroradiol 2003;24: 1237-39

22. Fanning NF, Willinsky RA, ter Brugge KG. Wall enhancement, edema, and hydrocephalus after endovascular coil occlusion of intradural cerebral aneurysms. J Neurosurg 2008;108:1074-86

23. Berge J, Tourdias T, Moreau JF, et al. Peri-aneurysmal brain inflammation after flow diversion treatment. AJNR Am J Neuroradiol 2011;32:1930-34 